\title{
Hypersymplectic geometry and supersymmetric solutions in $(t, s)$ 5D supergravity
}

\author{
W. A. Sabra® \\ Physics Department, American University of Beirut, \\ P.O. Box 11-0236, Riad El-Solh, Beirut 1107 2020, Lebanon
}

(Received 9 June 2021; accepted 2 July 2021; published 9 August 2021)

Relying on the method of spinorial geometry, purely bosonic supersymmetric solutions in $N=2$, five-dimensional supergravity theories coupled to vector multiplets in all space-time signatures are found. Explicit examples of some new solutions are presented.

DOI: $10.1103 /$ PhysRevD.104.046012

\section{INTRODUCTION}

The five-dimensional $N=2$ supergravity theories with Lorentzian signature coupled to vector multiplets was constructed many years ago in [1]. In recent years, there has been some interest in supergravity theories in various space-time signatures. The Euclidean versions of the supergravity theories of [1] were considered in [2], where it was demonstrated that the Lagrangian of the Euclidean theory has the kinetic terms of the gauge fields with the nonconventional sign. Euclidean $N=2$ theories in four dimensions were first considered in [3-6]. The Euclidean four-dimensional $N=2$ supergravity theories were obtained as dimensional reductions of $N=2, D=5$ supergravity theories on a timelike circle. The reduction of the five-dimensional Euclidean theory on a circle produces Euclidean $N=2$ four-dimensional supergravity with the nonconventional signs of the gauge fields kinetic terms. The four-dimensional Euclidean supergravity theory with vector and hypermultiplets was also be obtained via the dimensional reduction of Euclidean ten-dimensional type IIA supergravity over a Calabi-Yau threefold, $\mathrm{CY}_{3}$ [7]. A class of Lorentzian five-dimensional $N=2$ supergravity theories constructed in [1] is obtainable via the dimensional reduction of the standard eleven-dimensional supergravity [8] on a $\mathrm{CY}_{3}$ [9]. Recently, in [10], $N=2$ four and five-dimensional supergravity theories in space-time signatures $(t, s)$, where $t$ and $s$ are respectively the number of time and spatial dimensions, were constructed by reducing Hull's elevendimensional supergravity [11] on $\mathrm{CY}_{3}$. For a detailed analysis on supersymmetry algebras in arbitrary space-time dimension and signature we refer the reader to [12].

Published by the American Physical Society under the terms of the Creative Commons Attribution 4.0 International license. Further distribution of this work must maintain attribution to the author(s) and the published article's title, journal citation, and DOI. Funded by SCOAP ${ }^{3}$.
The eleven dimensional supergravity theories of Hull with space-time signatures $(1,10),(5,6)$ and $(9,2)$ have actions with the standard conventional sign for the 3 -form gauge kinetic term. The mirror theories with signatures $(10,1),(6,5)$, and $(2,9)$ all have the non-conventional sign for the 3 -form gauge fields kinetic terms. In the reduction of the theories with signatures $(1,10),(5,6)$, and $(2,9)$, the $C Y_{3}$ is taken to be of signature $(0,6)$. For the reduction of theories with signatures $(10,1),(6,5)$, and $(9,2)$, the $C Y_{3}$ is of signature $(6,0)$.

By employing the methods of [13], a systematic classification of supersymmetric solutions of the $(1,4)$ fivedimensional minimal supergravity was given in [14]. In this approach, the existence of at least one Killing spinor is assumed and differential forms as bilinears in terms of this spinor are constructed. The algebraic and differential constraints satisfied by the bilinears can be used to fix the solution of the space-time metric in addition to the bosonic fields of the supersymmetric solution. It was found in [14] that half-supersymmetric solutions with time-like Killing vectors have a four-dimensional base space given by a hyperKähler manifold. These findings for the time-like solutions were generalized to supergravity theories coupled to arbitrary many Abelian vector multiplets in [15] where also a uniqueness theorem for asymptotically flat supersymmetric black holes with regular horizons was given.

The goal of our present work is the generalization of the results unique to all $N=2$, five-dimensional supergravity theories coupled to vector multiplets in all space-time signatures. The Killing spinor equations shall be analysed using the spinorial geometry methods which were first employed in the analysis of supersymmetric solutions in ten and eleven dimensions in [16]. Spinorial geometry [17] has been very useful and efficient in the classifications of solutions with various fractions of supersymmetry in all space-time dimensions [18].

We organize our work as follows. In Sec. II, a review of some of the basic properties of the ungauged 
five-dimensional supergravity coupled to arbitrary many vector multiplets is given. Section III contains the analysis of supersymmetric solutions where the set of rules for the construction of these solutions is given. Some examples and a summary are given in Sec. IV.

\section{II. $(t, s)$ FIVE-DIMENSIONAL SUPERGRAVITY}

Ignoring hypermultiplets, the bosonic action of the theory for all $N=2, D=5$ supergravity contains the gravity multiplet and vector multiplets and is given by $[1,10]$

$$
\begin{aligned}
S_{5}= & \int_{M_{5}} \frac{1}{2} R \hat{*} 1-\frac{1}{2} Q_{I J}(X) d X^{I} \wedge * d X^{J} \\
& +\frac{\kappa^{2}}{2} Q_{I J}(X) F^{I} \wedge \hat{*} F^{J}-\frac{1}{12} C_{I J K} A^{I} \wedge F^{J} \wedge F^{K}
\end{aligned}
$$

where $C_{I J K}$ are real constants symmetric in $I, J, K$. We have $\kappa^{2}=-1$, for signature $(1,4),(5,0)$ and $(3,2)$ theories and $\kappa^{2}=1$ for signature $(4,1),(0,5)$, and $(2,3)$. Here $F^{I}$ are twoforms representing the gauge fields. The information about the theory is encoded in the cubic prepotential which describes very special geometry

$$
\mathcal{V}=\frac{1}{6} C_{I J K} X^{I} X^{J} X^{K}=1,
$$

$X^{I}$ being the very special coordinates, functions of the $n$ real scalar fields belonging to the vector multiplets.

The gauge coupling metric can be derived from the prepotential and is given

$Q_{I J}=-\frac{1}{2}\left(\partial_{X^{I}} \partial_{X^{J}}(\ln \mathcal{V})\right)_{\mathcal{V}=1}=\frac{1}{2}\left(9 X_{I} X_{J}-C_{I J K} X^{K}\right)$,

where the dual fields $X_{I}$ are defined by

$$
X_{I}=\frac{1}{6} C_{I J K} X^{J} X^{K}
$$

We also have the useful relations

$$
Q_{I J} X^{J}=\frac{3}{2} X_{I}, \quad Q_{I J} d X^{J}=-\frac{3}{2} d X_{I} .
$$

The Killing spinor equations associated with the above theories are given

$$
\left[\nabla_{\mu}+\frac{\kappa}{8} H_{\rho \sigma}\left(\Gamma_{\mu} \Gamma^{\rho \sigma}-6 \delta_{\mu}^{\rho} \Gamma^{\sigma}\right)\right] \epsilon=0
$$

and

$$
\left(\kappa G_{\mu \nu}^{I} \Gamma^{\mu \nu}-2 \partial_{\mu} X^{I} \Gamma^{\mu}\right) \epsilon=0
$$

where

$$
\begin{aligned}
G_{\mu \nu}^{I} & =F_{\mu \nu}^{I}-X^{I} X_{J} F_{\mu \nu}^{J}, \\
\nabla_{\mu} & =\partial_{\mu}+\frac{1}{4} \omega_{\mu, \rho \sigma} \Gamma^{\rho \sigma}, \\
H_{\mu \nu} & =X_{I} F_{\mu \nu}^{I} .
\end{aligned}
$$

Here $\Gamma_{\mu}$ are Dirac matrices and $\omega_{\mu, \rho \sigma}$ are the spin connections. For the supergravity theories with space-time signature $(1,4),(3,2)$, and $(5,0)$, we have $\kappa=-i$. For the supergravity theories with space-time signatures $(4,1)$, $(2,3)$, and $(0,5)$, we have $\kappa=1$.

\section{SUPERSYMMETRIC SOLUTIONS}

In what follows, we find solutions admitting Killing spinors through the analysis of the Killing spinor equations (2.6) and (2.7) using spinorial geometry methods. We take the Dirac spinors to be the space of complex forms on $\mathbb{R}^{2}$ spanned over $\mathbb{C}$ by $1, e_{1}, e_{2}$ and $e_{12}=e_{1} \wedge e_{2}$. To proceed in the analysis of solutions admitting Killing spinors, we start by writing our metric solutions in the form

$$
\begin{aligned}
d s_{5}^{2} & =\kappa^{2}\left(\mathbf{e}^{5}\right)^{2}+\eta_{\alpha \bar{\beta}} \mathbf{e}^{\alpha} \mathbf{e}^{\bar{\beta}} \\
& =\kappa^{2}\left(\mathbf{e}^{5}\right)^{2}+2\left(\kappa_{1}^{2} \mathbf{e}^{1} \mathbf{e}^{\overline{1}}+\kappa_{2}^{2} \mathbf{e}^{2} \mathbf{e}^{\overline{2}}\right)
\end{aligned}
$$

where $\kappa^{2}, \kappa_{1}^{2}$ and $\kappa_{2}^{2}$ are chosen to be \pm 1 , depending on the space-time signature of the considered theory. For example, if we are considering the supergravity theories with $(2,3)$ signature, we take $\kappa^{2}=\kappa_{1}^{2}=1, \kappa_{2}^{2}=-1$ or alternatively $\kappa^{2}=\kappa_{2}^{2}=1, \kappa_{1}^{2}=-1$.

The action of the $\Gamma$-matrices on spinors is given by

$$
\begin{aligned}
\Gamma_{1} & =\kappa_{1} \sqrt{2} e^{1} \wedge, \quad \Gamma_{\overline{1}}=\kappa_{1} \sqrt{2} i_{e^{1}}, \\
\Gamma_{2} & =\sqrt{2} \kappa_{2} e^{2} \wedge, \quad \Gamma_{\overline{2}}=\sqrt{2} \kappa_{2} i_{e^{2}}, \\
\Gamma_{5} 1 & =\kappa 1, \quad \Gamma_{5} e_{1}=-\kappa e_{1}, \\
\Gamma_{5} e_{2} & =-\kappa e_{2}, \quad \Gamma_{5} e_{12}=\kappa e_{12} .
\end{aligned}
$$

We shall find solutions for the Killing spinor $\epsilon=f 1$. This Killing spinor orbit corresponds to timelike solutions in the standard supergravity models with signature $(1,4)$. Plugging $\epsilon=f 1$ in the Killing spinor equation (2.6), we obtain the following conditions 


$$
\begin{array}{rlrl}
\partial_{\alpha} \log f+\frac{1}{2} \omega_{\alpha, \mu}{ }^{\mu}-\frac{3}{4} H_{\alpha 5} & =0, & H_{\alpha \beta} & =-2 \kappa^{2} \omega_{5, \alpha \beta}, \\
\partial_{\bar{\alpha}} \log f+\frac{1}{2} \omega_{\bar{\alpha}, \mu}{ }^{\mu}-\frac{1}{4} H_{\bar{\alpha} 5} & =0, & H_{\alpha 5} & =2 \partial_{\alpha} \log f, \\
\partial_{5} \log f+\frac{1}{2} \omega_{5, \mu}{ }^{\mu}+\frac{1}{4} \kappa^{2} H_{\mu}{ }^{\mu} & =0, & H_{\alpha \bar{\beta}} & =\frac{2}{3} \kappa^{2}\left(\omega_{\alpha, \bar{\beta} 5}-\eta_{\alpha \bar{\beta}} \omega_{5, \mu}{ }^{\mu}\right), \\
H_{\mu}{ }^{\mu} & =-2 \kappa^{2} \omega_{5, \mu}{ }^{\mu} .
\end{array}
$$$$
\kappa^{2} \omega_{1, \overline{1} 5}+\frac{1}{2} \kappa_{1}^{2} H_{\mu}{ }^{\mu}-\frac{3}{2} H_{1 \overline{1}}=0,
$$$$
\kappa^{2} \omega_{2, \overline{2} 5}+\frac{1}{2} \kappa_{2}^{2} H_{\mu}{ }^{\mu}-\frac{3}{2} H_{2 \overline{2}}=0,
$$$$
\kappa^{2} \omega_{2, \overline{1} 5}-\frac{3}{2} H_{2 \overline{1}}=0,
$$$$
\kappa^{2} \omega_{1, \overline{2} 5}-\frac{3}{2} H_{1 \overline{2}}=0 \text {, }
$$$$
\omega_{\overline{2}, \overline{1} 5}-\frac{1}{2} \kappa^{2} H_{\overline{2} \overline{1}}=0,
$$$$
\omega_{\overline{1}, \overline{2} 5}-\frac{\kappa^{2}}{2} H_{\overline{1} \overline{2}}=0 \text {, }
$$$$
\omega_{1, \overline{1} \overline{2}}+\frac{1}{2} \kappa_{1}^{2} H_{\overline{2} 5}=0,
$$$$
\omega_{2, \overline{1} \overline{2}}-\frac{1}{2} \kappa_{2}^{2} H_{\overline{1} 5}=0 \text {, }
$$$$
\omega_{\alpha, \beta \gamma}=0
$$$$
\omega_{\alpha, \alpha 5}=0 \text {, }
$$$$
\omega_{5, \overline{1} 5}-\kappa^{2} H_{5 \overline{1}}=0,
$$$$
\omega_{5, \overline{2} 5}-\kappa^{2} H_{5 \overline{2}}=0 \text {, }
$$$$
\omega_{5, \overline{1} \overline{2}}+\frac{1}{2} \kappa^{2} H_{\overline{1} \overline{2}}=0 .
$$

The analysis of this linear system of equations implies the following conditions

$$
\begin{aligned}
\partial_{5} f & =0, \\
\omega_{5,5 \alpha}-2 \kappa^{2} \partial_{\alpha} \log f & =0, \\
\omega_{5, \alpha \beta}+\omega_{\alpha, \beta 5} & =0, \\
\omega_{\overline{\bar{\beta}, \alpha 5}}+\omega_{\alpha, \bar{\beta} 5} & =0, \\
\omega_{5, \mu}{ }^{\mu}-\omega_{\mu, 5^{\mu}} & =0, \\
\omega_{\alpha, \mu}{ }^{\mu}-\partial_{\alpha} \log f & =0, \\
\omega_{\alpha, \beta \gamma} & =0, \\
\omega_{\alpha, \bar{\mu} \bar{\nu}}+\eta_{\alpha \bar{\mu}} \partial_{\bar{\nu}} \log f-\eta_{\alpha \bar{\nu}} \partial_{\bar{\mu}} \log f & =0,
\end{aligned}
$$

and
The analysis of (2.7) gives the conditions

$$
\begin{aligned}
F_{\mu}^{I}{ }^{\mu} & =X^{I} H_{\mu}{ }^{\mu}, \\
F_{5 \alpha}^{I} & =X^{I} H_{5 \alpha}-\partial_{\alpha} X^{I}, \\
F_{\alpha \beta}^{I} & =X^{I} H_{\alpha \beta}, \\
\partial_{5} X^{I} & =0 .
\end{aligned}
$$

To proceed, we define the 1-form

$$
V=f^{2} \mathbf{e}^{5}
$$

and introduce the coordinate $\tau$ such that the dual vector field is given by $\kappa^{2} f^{2} \frac{\partial}{\partial \tau}$. The first four conditions in (3.3) provide the necessary and sufficient conditions for $V$ to define a Killing vector. Also those conditions imply that

$$
\mathcal{L}_{V} \mathbf{e}^{5}=0
$$

Furthermore one finds

$$
\begin{aligned}
& \mathcal{L}_{V} \mathbf{e}^{1}=-\kappa_{1}^{2} f^{2}\left[\left(\omega_{5, \overline{1} 1}-\omega_{1, \overline{1} 5}\right) \mathbf{e}^{1}+\left(\omega_{5, \overline{1} 2}-\omega_{2, \overline{1} 5}\right) \mathbf{e}^{2}\right] \\
& \mathcal{L}_{V} \mathbf{e}^{2}=-\kappa_{2}^{2} f^{2}\left[\left(\omega_{5, \overline{2} 1}-\omega_{1, \overline{2} 5}\right) \mathbf{e}^{1}+\left(\omega_{5, \overline{2} 2}-\omega_{2, \overline{2} 5}\right) \mathbf{e}^{2}\right]
\end{aligned}
$$

By making an appropriate gauge transformation as discussed in [19], we can set

$$
\mathcal{L}_{V} \mathbf{e}^{\alpha}=0 .
$$

We can choose coordinates such that

$$
\mathbf{e}^{5}=f^{2}(d \tau+w), \quad \mathbf{e}^{\alpha}=f^{-1} \mathbf{E}^{\alpha} .
$$

where the function $f$, the one-form $w$ and $\mathbf{E}^{\alpha}$ are all independent of the coordinate $\tau$. At this stage, we define the following three two-forms:

$$
\begin{aligned}
& \mathbf{J}_{1}=\mathbf{E}^{1} \wedge \mathbf{E}^{2}+\mathbf{E}^{\overline{1}} \wedge \mathbf{E}^{\overline{2}}, \\
& \mathbf{J}_{2}=-i\left(\mathbf{E}^{1} \wedge \mathbf{E}^{2}-\mathbf{E}^{\overline{1}} \wedge \mathbf{E}^{\overline{2}}\right), \\
& \mathbf{J}_{3}=i\left(\kappa_{1}^{2} \mathbf{E}^{1} \wedge \mathbf{E}^{\overline{1}}+\kappa_{2}^{2} \mathbf{E}^{2} \wedge \mathbf{E}^{\overline{2}}\right) .
\end{aligned}
$$

It can be shown that

$$
d \mathbf{J}_{1}=d \mathbf{J}_{2}=d \mathbf{J}_{3}=0
$$


provided

$$
\Omega_{\alpha, \bar{\mu} \bar{\nu}}=0, \quad \Omega_{\alpha, \beta \gamma}=0, \quad \Omega_{\alpha, \mu}{ }^{\mu}=0,
$$

where $\Omega$ represent the spin connections of the base manifold with vielbeins $\mathbf{E}^{\alpha}$. In fact, the conditions in (3.12) are implied by the last three conditions of (3.3). Moreover, $\mathbf{J}_{i}, i=1,2,3$, are covariantly constant twoforms on the base manifold. They also satisfy the following algebra

$\mathbf{J}_{1}^{2}=\mathbf{J}_{2}^{2}=-\kappa_{1}^{2} \kappa_{2}^{2}, \quad \mathbf{J}_{3}^{2}=-1, \quad \mathbf{J}_{1} \mathbf{J}_{2}=-\mathbf{J}_{2} \mathbf{J}_{1}=-\kappa_{1}^{2} \kappa_{2}^{2} \mathbf{J}_{3}$.

For $\kappa_{1}^{2} \kappa_{2}^{2}=-1$, relevant for theories with space-time signatures $(2,3)$ and $(3,2)$, the algebra (3.13) is that of paraquaternions or the so-called split quaternions [20]. We shall refer to the base manifold with such a structure as hypersymplectic [21]. For the cases with $\kappa_{1}^{2}=\kappa_{2}^{2}= \pm 1$, relevant for space-time signatures $(1,4),(4,1),(5,0)$ and $(0,5)$, the algebra (3.13) defines the algebra of quaternions.

We now turn back to the analysis of the gauge fields. Using (3.4), we have

$$
\begin{aligned}
H= & -2 \kappa^{2} \omega_{5, \alpha \beta} \mathbf{e}^{\alpha} \wedge \mathbf{e}^{\beta}+2 \partial_{\alpha} \log f \mathbf{e}^{\alpha} \wedge \mathbf{e}^{5} \\
& +\frac{2}{3} \kappa^{2}\left(\omega_{\alpha, \bar{\beta} 5}-\eta_{\alpha \bar{\beta}} \omega_{5, \mu}^{\mu}\right) \mathbf{e}^{\alpha} \wedge \mathbf{e}^{\bar{\beta}} .
\end{aligned}
$$

Noting that

$$
\begin{aligned}
\kappa^{2} d \mathbf{e}^{5}= & -2 \kappa^{2} \mathbf{e}^{5} \wedge d \log f \\
& +2\left(\omega_{1,5 \overline{1}} \mathbf{e}^{\overline{1}} \wedge \mathbf{e}^{1}+\omega_{2,5 \overline{2}} \mathbf{e}^{\overline{2}} \wedge \mathbf{e}^{2}\right) \\
& +2\left(\omega_{\overline{1}, 52} \mathbf{e}^{2}+\omega_{\overline{1}, 52} \mathbf{e}^{\overline{2}}\right) \wedge \mathbf{e}^{\overline{1}} \\
& +2\left(\omega_{1,52} \mathbf{e}^{2}+\omega_{1,5 \overline{2}} \mathbf{e}^{\overline{2}}\right) \wedge \mathbf{e}^{1} .
\end{aligned}
$$

We obtain

$$
\begin{aligned}
H-d \mathbf{e}^{5}= & -\frac{2}{3} \kappa^{2}\left(\omega_{1,5 \overline{1}} \mathbf{e}^{\overline{1}} \wedge \mathbf{e}^{1}+\omega_{2,5 \overline{2}} \mathbf{e}^{\overline{2}} \wedge \mathbf{e}^{2}\right. \\
& \left.+2 \omega_{1,5 \overline{2}} \mathbf{e}^{\overline{2}} \wedge \mathbf{e}^{1}+2 \omega_{\overline{1}, 52} \mathbf{e}^{2} \wedge \mathbf{e}^{\overline{1}}\right) \\
& -\frac{2}{3} \kappa^{2} \kappa_{1}^{2} \kappa_{2}^{2}\left(\omega_{5,2 \overline{2}} \mathbf{e}^{1} \wedge \mathbf{e}^{\overline{1}}+\omega_{5,1 \overline{1}} \mathbf{e}^{2} \wedge \mathbf{e}^{\overline{2}}\right) .
\end{aligned}
$$

The right hand of the above equation can be expressed in terms of the self-dual part of $d w$, and we have

$$
H=d \mathbf{e}^{5}-\frac{f^{2}}{3}(d w+* d w),
$$

where our orientation is such that $\epsilon_{1 \overline{1} 2 \overline{2}}=\kappa_{1}^{2} \kappa_{2}^{2}$. If we write

$$
f^{2} d w=G_{+}+G_{-},
$$

then we have

$$
H=d \mathbf{e}^{5}+\Psi
$$

with

$$
\Psi=-\frac{2}{3} G_{+}
$$

and thus $\Psi$ is a self-dual 2-form on the base manifold. Using (3.5), we find

$$
F^{I}=d\left(X^{I} \mathbf{e}^{5}\right)+\Psi^{I}
$$

where $\Psi=X_{I} \Psi^{I}$. The Bianchi identity then implies

$$
d \Psi^{I}=0
$$

and thus $\Psi^{I}$ are harmonic self-dual 2-forms on the base manifold with metric $d s_{4}^{2}=\eta_{\alpha \bar{\beta}} \mathbf{e}^{\alpha} \mathbf{e}^{\bar{\beta}}$. Turning to Maxwell equations

$$
d\left(Q_{I J} * F^{J}\right)=\frac{\kappa^{2}}{4} C_{I J K} F^{J} \wedge F^{K}
$$

we obtain after some calculation

$$
\nabla^{2}\left(f^{-2} X_{I}\right)=-\frac{\kappa^{2}}{6} C_{I J K} \Psi^{J} \cdot \Psi^{K}
$$

where the Laplacian is for the metric $d s_{4}^{2}=\eta_{\alpha \bar{\beta}} \mathbf{E}^{\alpha} \mathbf{E}^{\bar{\beta}}$ and we have the convention that for two $p$-forms $\alpha$ and $\beta$, we have

$$
\alpha . \beta=\frac{1}{p !} \alpha_{n_{1} \ldots n_{2}} \beta^{n_{1} \ldots n_{2}} .
$$

Finally we note that the integrability conditions for the Killing spinor equations together with imposing the Bianchi identity and the equations of motion for the gauge fields guarantee that all the equations of motion are satisfied.

\section{EXAMPLES AND DISCUSSION}

In [14] solutions for the minimal case (no vector multiplets) with base space $\mathbb{R}^{4}$ were constructed. The four dimensional base metric can be expressed in terms of the left or right invariant forms of $S U(2)$ given in terms of Euler angles. The right invariant one forms are given by

$$
\begin{aligned}
& \sigma_{1}=\sin \phi d \theta-\cos \phi \sin \theta d \psi, \\
& \sigma_{2}=\cos \phi d \theta+\sin \theta \sin \phi d \psi, \quad \sigma_{3}=d \phi+\cos \theta d \psi
\end{aligned}
$$


and the left invariant ones are given by

$$
\begin{aligned}
& \chi_{1}=-\sin \psi d \theta+\cos \psi \sin \theta d \phi, \\
& \chi_{2}=\cos \psi d \theta+\sin \theta \sin \psi d \phi, \\
& \chi_{3}=d \psi+\cos \theta d \phi
\end{aligned}
$$

satisfying

$d \sigma_{i}=-\frac{1}{2} \epsilon_{i j k} \sigma_{j} \wedge \sigma_{k}, \quad d \chi_{i}=\frac{1}{2} \epsilon_{i j k} \chi_{j} \wedge \chi_{k}$.

In terms of these forms, the flat four-dimensional metric can be written in the form

$$
\begin{aligned}
d s_{4}^{2} & =d r^{2}+\frac{r^{2}}{4}\left(\sigma_{1}^{2}+\sigma_{2}^{2}+\sigma_{3}^{2}\right) \\
& =d r^{2}+\frac{r^{2}}{4}\left(\chi_{1}^{2}+\chi_{2}^{2}+\chi_{3}^{2}\right) \\
& =d r^{2}+\frac{r^{2}}{4}\left(d \theta^{2}+\sin ^{2} d \phi^{2}+(d \psi+\cos \theta d \phi)^{2}\right) .
\end{aligned}
$$

Defining

$$
\mathbf{E}^{1}=\frac{1}{\sqrt{2}}\left(e^{0}+i e^{3}\right) \quad \mathbf{E}^{2}=\frac{1}{\sqrt{2}}\left(e^{2}+i e^{1}\right)
$$

with

$$
e^{0}=d r, \quad e^{1}=\frac{1}{2} r \sigma_{1}, \quad e^{2}=\frac{1}{2} r \sigma_{2} \quad e^{3}=\frac{1}{2} r \sigma_{3},
$$

the three complex structures are then can be given by

$$
\begin{aligned}
& \mathbf{J}_{1}=e^{0} \wedge e^{1}-e^{2} \wedge e^{3}=\frac{1}{4} d\left(r^{2} \sigma_{1}\right), \\
& \mathbf{J}_{2}=e^{0} \wedge e^{2}+e^{1} \wedge e^{3}=\frac{1}{4} d\left(r^{2} \sigma_{2}\right), \\
& \mathbf{J}_{3}=e^{0} \wedge e^{3}-e^{1} \wedge e^{2}=\frac{1}{4} d\left(r^{2} \sigma_{3}\right) .
\end{aligned}
$$

For solutions with neutral flat base space, we can express the four-dimensional base metric in terms of the forms

$$
\begin{aligned}
& \sigma_{1}^{\prime}=\sin \phi d \theta-\cos \phi \sinh \theta d \psi, \\
& \sigma_{2}^{\prime}=\cos \phi d \theta+\sinh \theta \sin \phi d \psi, \\
& \sigma_{3}^{\prime}=d \phi+\cosh \theta d \psi
\end{aligned}
$$

or

$$
\begin{aligned}
& \chi_{1}^{\prime}=-\sin \psi d \theta+\cos \psi \sinh \theta d \phi, \\
& \chi_{2}^{\prime}=\cos \psi d \theta+\sinh \theta \sin \psi d \phi, \\
& \chi_{3}^{\prime}=d \psi+\cosh \theta d \phi
\end{aligned}
$$

satisfying

$$
d \sigma_{i}^{\prime}=-\frac{1}{2} f_{i j k} \sigma_{j}^{\prime} \wedge \sigma_{k}^{\prime}, \quad d \chi_{i}^{\prime}=\frac{1}{2} f_{i j k} \chi_{j}^{\prime} \wedge \chi_{k}^{\prime}
$$

where $f_{i j k}$ are the structure constants of $S O(2,1)$. The metric takes the form

$$
\begin{aligned}
d s_{4}^{2} & =d r^{2}+\frac{r^{2}}{4}\left(-\sigma_{1}^{\prime 2}-\sigma_{2}^{\prime 2}+\sigma_{3}^{\prime 2}\right) \\
& =d r^{2}+\frac{r^{2}}{4}\left(-\chi_{1}^{\prime 2}-\chi_{2}^{\prime 2}+\chi_{3}^{\prime 2}\right) \\
& =d r^{2}+\frac{r^{2}}{4}\left(-d \theta^{2}-\sinh ^{2} \theta d \phi^{2}+(d \psi+\cosh \theta d \phi)^{2}\right) .
\end{aligned}
$$

In this case the three two-forms satisfying the hypersymplectic algebra can be given by

$$
\mathbf{J}_{i}=\frac{1}{4} d\left(r^{2} \sigma_{i}^{\prime}\right), \quad i=1,2,3
$$

As an example, we consider the STU model with spacetime signatures $(2,3)$ and $(3,2)$ described by the prepotential $\mathcal{V}=X^{1} X^{2} X^{3}$. The solutions with signatures $(1,4)$ were considered in $[15,22]$. The metric is given by the general form

$$
\begin{aligned}
d s_{5}^{2}= & \kappa^{2} f^{4}(d \tau+w)^{2}+f^{-2}\left[d r^{2}+\frac{r^{2}}{4}\left(-d \theta^{2}-\sinh ^{2} \theta d \phi^{2}\right.\right. \\
& \left.\left.+(d \psi+\cosh \theta d \phi)^{2}\right)\right]
\end{aligned}
$$

where $\kappa^{2}=1$ for solutions with $(2,3)$ signature and $\kappa^{2}=-1$ for solutions with $(3,2)$ signature. We consider the simple case with vanishing $\Psi^{I}$ in (3.23) which implies that $f^{-2} X_{I}$ are given in terms of harmonic functions $H_{I}$ on the base space and we obtain

$$
\begin{aligned}
f^{-6} & =H_{1} H_{2} H_{3} \quad X^{1}=\left(\frac{H_{3} H_{2}}{H_{1}^{2}}\right)^{1 / 3}, \\
X^{2} & =\left(\frac{H_{3} H_{1}}{H_{2}^{2}}\right)^{1 / 3}, \quad X^{3}=\left(\frac{H_{2} H_{1}}{H_{3}^{2}}\right)^{1 / 3} .
\end{aligned}
$$

The gauge fields are given by

$$
F^{I}=d\left(X^{I} f^{2}(d \tau+w)\right) .
$$


As $G_{+}=0$, we obtain from (3.18) that $d w$ is anti-self-dual and we can set

$$
w=\frac{J}{r^{2}}(d \phi+\cosh \theta d \psi)
$$

with a constant $J$.

One can also consider solutions with neutral base given by an analytic continuation of the Eguchi-Hanson metric given by [23]

$$
d s_{4}^{2}=W^{-1} d r^{2}+\frac{r^{2}}{4}\left(-\sigma_{1}^{\prime 2}-\sigma_{2}^{\prime 2}+W \sigma_{3}^{\prime 2}\right)
$$

with

$$
W=1-\frac{a^{4}}{r^{4}} .
$$

In this case, the hypersymplectic structure is defined by

$$
\mathbf{J}_{i}=d\left(\frac{1}{4} r^{2} W^{1 / 2} \sigma_{i}^{\prime}\right) .
$$

One can also have analytic continuations of the general hyper-Kähler $N$-multi-centered Gibbons-Hawking metrics which admit triholomorphic Killing vector field [24,25] and obtain hypersymplectic metrics. Recall that these metrics are described by

$$
\begin{aligned}
d s^{2} & =V^{-1}\left(d x^{4}+\theta\right)+V\left(\left(d x^{1}\right)^{2}+\left(d x^{2}\right)^{2}+\left(d x^{3}\right)^{2}\right) \\
\nabla \times \theta & =\nabla V, \quad V=\eta+\sum_{i=1}^{N} \frac{\varrho}{\left|x-x_{i}\right|}
\end{aligned}
$$

where $\eta$ and $\varrho$ are constants. The triholomorphic Killing vector is $\partial_{x^{4}}$ and $\theta=\theta_{i} d x^{i}$. For $\eta=1$ and $N=0$, we obtain flat space and for $\eta=0$ and $N=1$, we obtain Eguchi-Hanson metric. One can analytically continue the metrics (3.43) and obtain hypersymplectic metrics. For example we can consider the metrics

$$
d s^{2}=V^{-1}\left(d x^{4}+\theta\right)+V\left(-\left(d x^{1}\right)^{2}-\left(d x^{2}\right)^{2}+\left(d x^{3}\right)^{2}\right)
$$

with the hypersymplectic structure given by

$$
\begin{aligned}
& \mathbf{J}_{1}=\left(d x^{4}+\theta\right) \wedge d x^{1}-V d x^{2} \wedge d x^{3}, \\
& \mathbf{J}_{2}=\left(d x^{4}+\theta\right) \wedge d x^{2}-V d x^{3} \wedge d x^{1}, \\
& \mathbf{J}_{3}=\left(d x^{4}+\theta\right) \wedge d x^{3}+V d x^{1} \wedge d x^{2} .
\end{aligned}
$$

All the solutions considered in [14] which included generalizations of BMPV black hole solutions [26], rotating Eguchi-Hanson and Taub-NUT solutions and solutions with Gibbons-Hawking base space can be analytically continued to obtain solutions with neutral bases. The continued hypersymplectic manifold will inherit the Killing fields of the Euclidean metric [23]. However it must be emphasized that neutral manifolds are less rigid than Riemannian manifolds. For example, Killing vectors with zero norms can not exist in the Riemannian case. Not all neutral hypersymplectic metrics can be obtained by analytic continuations.

In general, hypersymplectic metrics can be written in terms of one function in the form

$$
\begin{aligned}
d s^{2}= & \frac{\partial^{2} Y}{\partial x \partial w} d x d w+\frac{\partial^{2} Y}{\partial y \partial z} d y d z \\
& +\frac{\partial^{2} Y}{\partial y \partial w} d y d w+\frac{\partial^{2} Y}{\partial x \partial z} d x d z
\end{aligned}
$$

where the function $Y$ satisfies the so-called the first Heavenly equation $[27,28]$

$$
\frac{\partial^{2} Y}{\partial x \partial w} \frac{\partial^{2} Y}{\partial y \partial z}-\frac{\partial^{2} Y}{\partial y \partial w} \frac{\partial^{2} Y}{\partial x \partial z}=1 .
$$

An alternative representation of hypersymplectic metrics is given by

$$
\begin{aligned}
d s^{2}= & d y\left(d w-\frac{\partial^{2} S}{\partial x^{2}} d y-\frac{\partial^{2} S}{\partial w \partial x} d z\right) \\
& -d z\left(d x+\frac{\partial^{2} S}{\partial w^{2}} d z+\frac{\partial^{2} S}{\partial w \partial x} d y\right)
\end{aligned}
$$

with $S$ satisfying the so-called second Heavenly equation [28]

$$
\frac{\partial^{2} S}{\partial w \partial y}-\frac{\partial^{2} S}{\partial z \partial x}+\frac{\partial^{2} S}{\partial w^{2}} \frac{\partial^{2} S}{\partial x^{2}}-\left(\frac{\partial^{2} S}{\partial x \partial w}\right)^{2}=0
$$

Many interesting four-dimensional hypersymplectic metrics with various types of Killing vectors such as null Killing vectors and conformal Killing vectors have been constructed (see for example [29,30]). Using the Heavenly equation formalism, a notable example of a class of noncompact metrics on the cotangent bundles of Riemann surfaces with genus $\geq 1$ was constructed in [31].

In what follows we shall consider the $(2,2)$ analogs of ppwaves [28] which in the notation of [29] take the form

$$
d s^{2}=d y(d w-Q(x, y) d y)-d z d x
$$

where $Q$ is an arbitrary function. These metrics have a null Killing vector $\partial_{w}$ which can be thought of as a neutral signature version of a triholomorphic Killing vector [30]. The metrics (3.49) were also considered in the context of twistors [32] and have also appeared in the analysis of [23] and in the classification of neutral solutions admitting Killing spinors [33]. Using our formalism we rewrite (3.49) in the form 


$$
d s^{2}=2\left(\mathbf{E}^{1} \mathbf{E}^{\overline{1}}-\mathbf{E}^{2} \mathbf{E}^{\overline{2}}\right)
$$

with

$$
\begin{aligned}
& \mathbf{E}^{1}=\frac{1}{2 \sqrt{2}}[d w+(1-Q) d y+i(d z-d x)] \\
& \mathbf{E}^{2}=\frac{1}{2 \sqrt{2}}[d w-(1+Q) d y+i(d z+d x)] .
\end{aligned}
$$

Then the hypersymplectic structures is expressed in terms of

$$
\begin{aligned}
& \mathbf{J}_{1}=\frac{1}{2}(d y \wedge d w-d z \wedge d x), \\
& \mathbf{J}_{2}=\frac{1}{2}(d y \wedge d z+(d w-Q d y) \wedge d x), \\
& \mathbf{J}_{3}=\frac{1}{2}(d y \wedge d z-(d w-Q d y) \wedge d x) .
\end{aligned}
$$

Again as an example we again consider solutions of the STU model with $G_{+}=0$. In this case we obtain

$$
\begin{aligned}
f^{-6} & =\mathcal{H}_{1} \mathcal{H}_{2} \mathcal{H}_{3} \quad X^{1}=\left(\frac{\mathcal{H}_{2} \mathcal{H}_{3}}{\mathcal{H}_{1}^{2}}\right)^{1 / 3}, \\
X^{2} & =\left(\frac{\mathcal{H}_{3} \mathcal{H}_{1}}{\mathcal{H}_{2}^{2}}\right)^{1 / 3}, \quad X^{3}=\left(\frac{\mathcal{H}_{2} \mathcal{H}_{1}}{\mathcal{H}_{3}^{2}}\right)^{1 / 3}
\end{aligned}
$$

where $\mathcal{H}_{i}$ are harmonic functions on the base space described by (3.49) which can be arbitrary functions of the coordinates $x$ and $y$. As $d w$ is anti-self-dual we can for example set

$$
d w=(d y \wedge d w-d z \wedge d x)
$$

In this paper we have considered a class of solutions admitting Killing spinors of five dimensional ungauged supergravity with Abelian vector multiplets. The base space of solutions with space-time signatures $(1,4),(4,1),(5,0)$ and $(0,5)$ are given in terms of hyper-Kähler manifolds. The solutions of the five dimensional theories with space-time signatures $(2,3)$ and $(3,2)$, the base manifold admits a hypersymplectic structure [21].

Hypersymplectic geometry has a very rich structure and not all hypersymplectic manifolds can be obtained from hyper-Kähler manifolds via analytic continuation. All the examples considered in $[14,15]$ can be analytically continued to obtain solutions with hypersymplectic base manifold. It would be of interest to construct many explicit solutions and generalize our results to gauged five-dimensional supergravity theories. We hope to report on this in a future publication.

\section{ACKNOWLEDGMENTS}

The work is supported in part by the National Science Foundation under Grant No. PHY-1620505. The author would like to thank M. Dunajski and J. Gutowski for useful discussions.
[1] M. Gunaydin, G. Sierra, and P. K. Townsend, The geometry of $N=2$ Maxwell-Einstein supergravity and Jordan algebras, Nucl. Phys. B242, 244 (1984).

[2] W. A. Sabra and O. Vaughan, Euclidean supergravity in five dimensions, Phys. Lett. B 760, 14 (2016).

[3] V. Cortes, C. Mayer, T. Mohaupt, and F. Saueressig, Special geometry of Euclidean supersymmetry I: Vector multiplets, J. High Energy Phys. 03 (2004) 028.

[4] V. Cortes, C. Mayer, T. Mohaupt, and F. Saueressig, Special geometry of Euclidean supersymmetry II: Hypermultiplets and the c-map, J. High Energy Phys. 06 (2005) 025.

[5] V. Cortes and T. Mohaupt, Special geometry of Euclidean supersymmetry III: The local r-map, instantons and black holes, J. High Energy Phys. 07 (2009) 066.

[6] V. Cortés, P. Dempster, T. Mohaupt, and O. Vaughan, Special geometry of Euclidean supersymmetry IV: The local c-map, J. High Energy Phys. 10 (2015) 066.

[7] W. A. Sabra and O. Vaughan, 10D to 4D Euclidean supergravity over a Calabi-Yau three-fold, Classical Quantum Gravity 33, 1033 (2015).
[8] E. Cremmer, B. Julia, and J. Scherk, Supergravity theory in eleven-dimensions, Phys. Lett. 76B, 409 (1978).

[9] A. Ceresole, R. D'Auria, and S. Ferrara, 11-Dimensional supergravity compactified on Calabi-Yau threefolds, Phys. Lett. B 357, 76 (1995).

[10] W. A. Sabra, Special geometry and space-time signature, Phys. Lett. B 773, 191 (2017).

[11] C. M. Hull, Duality and the signature of space-time, J. High Energy Phys. 11 (1998) 017.

[12] L. Gall, Supersymmetry algebras in arbitrary dimension and signature, Ph.D. U. Liverpool, 2019.

[13] J. P. Gauntlett, N. W. Kim, D. Martelli, and D. Waldram, Fivebranes wrapped on SLAG three-cycles and related geometry, J. High Energy Phys. 11 (2001) 018; J. P. Gauntlett, D. Martelli, S. Pakis, and D. Waldram, G-structures and wrapped NS5-branes, Commun. Math. Phys. 247, 421 (2004).

[14] J. P. Gauntlett, J. B. Gutowski, C. M. Hull, S. Pakis, and H. S. Reall, Solutions of minimal supergravity in five dimensions, Classical Quantum Gravity 20, 4587 (2003). 
[15] J. B. Gutowski, Uniqueness of five-dimensional supersymmetric black hole, J. High Energy Phys. 08 (2004) 049.

[16] J. Gillard, U. Gran, and G. Papadopoulos, The spinorial geometry of supersymmetric backgrounds, Classical Quantum Gravity 22, 1033 (2005); U. Gran, J. Gutowski, and G. Papadopoulos, The spinorial geometry of supersymmetric IIb backgrounds, Classical Quantum Gravity 22, 2453 (2005).

[17] F. R. Harvey, Spinors and Calibrations (Academic Press, London, 1990).

[18] U. Gran, J. Gutowski, and G. Papadopoulos, Classification, geometry and applications of supersymmetric backgrounds, Phys. Rep. 749, 1 (2019).

[19] J. B. Gutowski and W. A. Sabra, HKT geometry and fake five dimensional supergravity, Classical Quantum Gravity 28, 175023 (2011).

[20] S. Ivanov and S. Zamkovy, Parahermitian and paraquaternionic manifolds, Differential Geometry and its Applications 23, 205 (2005); A. S. Dancer, H. R. Jorgensen, and A. F. Swann, Metric geometries over the split quaternions, arXiv:math/0412215.

[21] N. J. Hitchin, Hypersymplectic quotients, Acta Acad. Sci. Tauriensis 124, 169 (1990).

[22] W. A. Sabra, General BPS black holes in five dimensions, Mod. Phys. Lett. A 13, 239 (1998); A. H. Chamseddine and W. A. Sabra, Metrics admitting killing spinors in fivedimensions, Phys. Lett. B 426, 36 (1998).
[23] J. Barrett, G. W. Gibbons, M. J. Perry, C. N. Pope, and P. Ruback, Kleinian geometry and the $N=2$ superstring, Int. J. Mod. Phys. A 09, 1457 (1994).

[24] G. W. Gibbons and S. W. Hawking, Gravitational multiinstantons, Phys. Lett. 78B, 430 (1978).

[25] G. W. Gibbons and P. J. Ruback, The hidden symmetries of multicenter metrics, Commun. Math. Phys. 115, 267 (1988).

[26] J. C. Breckenridge, R. C. Myers, A. W. Peet, and C. Vafa, Dbranes and spinning black holes, Phys. Lett. B 391, 93 (1997).

[27] T. Sibata and K. Mornga, A complete and simpler treatment of wave geometry, Proc. Am. Math. Soc. 102, 157 (1988).

[28] J. F. Plebański, Some solutions of complex Einstein equations, J. Math. Phys. (N.Y.) 16, 2395 (1975).

[29] M. Dunajski and S. West, Anti-self-dual conformal structures in neutral signature, in Recent Developments in Pseudo-Riemannian Geometry, ESI-Series on Mathematics and Physics [arXiv:math/0610280].

[30] M. Dunajski and S. West, Anti-self-dual conformal structures with null killing vectors from projective structures, Commun. Math. Phys. 272, 85 (2007).

[31] H. Ooguri and C. Vafa, Self-duality and $N=2$ string magic, Mod. Phys. Lett. A 05, 1389 (1990); Geometry of $N=2$ strings, Nucl. Phys. B361, 469 (1991).

[32] R. S. Ward, A class of self-dual solutions of Einstein's equations, Proc. R. Soc. A 363, 289 (1978).

[33] J. B. Gutowski and W. A. Sabra, Real killing spinors in neutral signature, J. High Energy Phys. 11 (2019) 173. 